\title{
A BOUND FOR THE SPLITTING OF SMOOTH FANO POLYTOPES WITH MANY VERTICES
}

\author{
BENJAMIN ASSARF AND BENJAMIN NILL
}

\begin{abstract}
The classification of toric Fano manifolds with large Picard number corresponds to the classification of smooth Fano polytopes with large number of vertices. A smooth Fano polytope is a polytope that contains the origin in its interior such that the vertex set of each facet forms a lattice basis. Casagrande showed that any smooth $d$-dimensional Fano polytope has at most $3 d$ vertices. Smooth Fano polytopes in dimension $d$ with at least $3 d-2$ vertices are completely known. The main result of this paper deals with the case of $3 d-k$ vertices for $k$ fixed and $d$ large. It implies that there is only a finite number of isomorphism classes of toric Fano $d$-folds $X$ (for arbitrary $d$ ) with Picard number $2 d-k$ such that $X$ is not a product of a lower-dimensional toric Fano manifold and the projective plane blown up in three torus-invariant points. This verifies the qualitative part of a conjecture in a recent paper by the first author, Joswig, and Paffenholz.
\end{abstract}

\section{INTRODUCTION AND MAIN RESULTS}

Let us first recall the basic definitions. We refer to [19, 12] for more background. Let $N \cong \mathbb{Z}^{d}$ be a lattice with associated real vector space $N_{\mathbb{R}}:=N \otimes_{\mathbb{Z}} \mathbb{R}$ isomorphic to $\mathbb{R}^{d}$. A polytope $P$ is a convex, compact set in $N_{\mathbb{R}}$, its 0 -dimensional faces are called vertices, and its faces of codimension 1 are called facets. If every facet $F$ (of dimension $d-1$ ) of a $d$-dimensional polytope $P$ has exactly $d$ vertices (i.e., $F$ is a simplex), then $P$ is called simplicial. The polytope $P$ is called a lattice polytope if its vertices are lattice points (i.e., elements of $N$ ).

Definition 1. (i) A polytope $P \subset N_{\mathbb{R}}$ is called smooth Fano polytope, if $\triangleright P$ is a lattice polytope, and

$\triangleright P$ is full-dimensional and contains the origin 0 as an interior point, and

$\triangleright$ for each facet $F$ of $P$, the vertex set Vert $F$ is a lattice basis of $N$.

(ii) Two smooth Fano polytopes are lattice equivalent, if their vertex sets are in bijection by an affine-linear lattice automorphism.

Remark 2. We decided to keep the notion of a smooth Fano polytope in order to be consistent with existing literature. However, we remark that there exists also the definition of a smooth polytope as a lattice polytope with unimodular vertex cones. A smooth Fano polytope is not a smooth polytope (but its dual polytope is).

Note that any smooth Fano polytope $P$ is necessarily simplicial. In each dimension there exist only finitely many smooth Fano polytopes up to lattice equivalence (we refer to the survey [19]). In 2007, Øbro described an explicit classification algorithm

2010 Mathematics Subject Classification. 52B20, 14M25, 14J45.

Key words and phrases. toric Fano varieties, Fano polytopes, lattice polytopes, reflexive polytopes, smooth polytopes. 


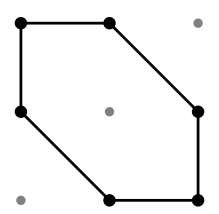

Figure 1. The smooth Fano polygon $P_{6}$ corresponding to the del Pezzo surface $S_{3}$

in each dimension $d$, see $[21,22]$. His implementation gave complete classifications of lattice equivalence classes of smooth Fano polytopes up to dimension 8 extending the previous existing classification results up to dimension 5 ; see $[2,25,4,24,16]$. Using a variant of Øbro's algorithm, the classification in dimension 9 was done by Lorenz and Paffenholz [17]. The large number of 8229721 smooth Fano polytopes in dimension 9 indicates that complete classifications in much higher dimensions are not feasible. This motivates to focus on finding sharp bounds on important invariants and to classify the extreme cases.

When considering smooth Fano polytopes with large number of vertices, one needs to start with dimension two. Let $P_{6}$ be the smooth Fano polygon with 6 vertices as given in Figure 1. This polygon has the maximal number of vertices possible in dimension 2 .

There is a natural direct sum operation on smooth Fano polytopes (also sometimes called free sum).

Definition 3. Given smooth Fano polytopes $Q \subset N_{\mathbb{R}}$ and $Q^{\prime} \subset N_{\mathbb{R}}^{\prime}$, we define their direct sum as

$$
Q \oplus Q^{\prime}=\operatorname{conv}\left(Q \times\{0\} \cup\{0\} \times Q^{\prime}\right) \quad \subset N_{\mathbb{R}} \times N_{\mathbb{R}}^{\prime} .
$$

Direct sums of smooth Fano polytopes are again smooth Fano polytopes. A smooth Fano polytope $P$ splits into $Q$ and $Q^{\prime}$ if $P$ is lattice equivalent to $Q \oplus Q^{\prime}$.

In 2006 Casagrande proved the following long-standing conjecture.

Theorem 4 (Casagrande [7]). Let $P$ be a d-dimensional smooth Fano polytope. Then $P$ has at most $3 d$ vertices, equality holds if and only if $d$ is even and $P$ is lattice equivalent to $\left(P_{6}\right)^{\oplus d / 2}$.

By now, smooth Fano polytopes with at least $3 d-2$ vertices are completely classified [23, 1]. In [1, Conjecture 9] it was conjectured that in order to classify smooth Fano polytopes $P$ of dimension $d$ with $\mid$ Vert $P \mid=3 d-k$ it is enough to do this up to dimension $3 k$. More precisely, if $d>3 k$, then $P$ should be a direct sum of $P_{6}$ and a lower-dimensional smooth Fano polytope. The goal of this paper is to verify the qualitative part of this conjecture.

Theorem 5. Let $P$ be a d-dimensional smooth Fano polytope with $3 d-k$ vertices and $d \geq 15 k^{2}+37 k+2$. Further let $f(d, k):=\left\lfloor\frac{d-15 k^{2}-37 k}{2}\right\rfloor$. Then $P$ is lattice equivalent to

$$
Q \oplus P_{6}^{\oplus f(d, k)}
$$

where $Q$ is a smooth Fano polytope of dimension $d-2 f(d, k)$.

Remark 6. Any smooth Fano polytope is a reflexive polytope, i.e., it contains the origin in its interior (as the only interior lattice point) and its dual polytope is also a 
lattice polytope (we refer to [3, 18]). Reflexive polytopes always appear as dual pairs. They correspond to Gorenstein toric Fano varieties and were introduced by Batyrev in 1994 to provide a combinatorial framework for constructing mirror symmetric pairs of Calabi-Yau hypersurfaces [3]. In fixed dimension only a finite number of reflexive polytopes exist up to lattice equivalence. Still, Haase and Melnikov showed that any lattice polytope is isomorphic to the face of some (possibly much higher-dimensional) reflexive polytope, [14]. Interestingly, it is conjectured that $P_{6}^{d / 2}$ has the maximal number of vertices of a reflexive polytope in even dimension; check [5]. Note that this is the dual reflexive polytope of $P_{6}^{\oplus d / 2}$.

1.1. The algebro-geometric viewpoint: background, applications, and conjectures. To make this paper accessible not only to combinatorialists but also to algebraic geometers, we decided to split the introduction. In this section we will use algebro-geometric language to describe the relevance in toric geometry, to discuss the equality case, and to present more detailed conjectures (all toric geometry statements can easily be translated into combinatorial ones). However, note that all proofs in the following sections will be purely combinatorial.

While smooth Fano polytopes are interesting peculiar classes of lattice polytopes, the main reason for their investigation originates in algebraic geometry. Toric Fano manifolds are among the most intensively studied classes of toric varieties. We refer to the surveys [11] and [19] and the references therein. By the toric dictionary (e.g. [10, Chapter 8]), any $d$-dimensional smooth Fano polytope $P$ corresponds one-to-one to a $d$-dimensional toric Fano manifold $X$. Two smooth Fano polytopes are lattice equivalent if and only if the corresponding toric Fano manifolds are isomorphic. In each dimension $d$, there exists only a finite number of toric Fano $d$-folds up to isomorphisms. As described in the previous section, they are completely classified up to dimension 9. Their large number in higher dimensions motivates the study of subclasses of special interest.

Let $X$ be a toric Fano $d$-fold corresponding to a smooth Fano polytope $P$ of dimension $d$. Then the Picard number $\rho_{X}$ of $X$ equals $\mid$ Vert $P \mid-d$. For instance, $P_{6}$ corresponds to the del Pezzo surface $S_{3}\left(\mathbb{P}^{2}\right.$ blown up in three torus-invariant points), note that $\rho_{S_{3}}=4$. A smooth Fano polytope $P$ splits into two smooth Fano polytopes if and only if $X$ is isomorphic to the product of the corresponding toric Fano manifolds.

Theorem 4, by Casagrande, implies that the Picard number $\rho_{X}$ of any toric Fano $d$-fold $X$ is bounded from above by $2 d$. Moreover, the equality case is only attained in even dimension $d$ by $\left(S_{3}\right)^{d / 2}$, the product of $d / 2$ copies of the del Pezzo surface $S_{3}$. In 2008, Øbro showed that if the Picard number equals $2 d-1$, then the variety $X$ is isomorphic to $\left(S_{3}\right)^{(d-2) / 2} \times S_{2}$ if $d$ is even, where $S_{2}$ is $\mathbb{P}^{2}$ blown up in two torus-invariant points, and $X$ is isomorphic to $\left(S_{3}\right)^{(d-3) / 2} \times Y$ if $d$ is odd, where $Y$ is one of two toric Fano 3-folds with $\rho_{Y}=5$. Recently, a complete classification of toric Fano $d$-folds $X$ with $\rho_{X}=2 d-2$ was given by Assarf, Joswig, Paffenholz. Their result gave rise to the following conjecture:

Conjecture 7 ([1, Conjecture 9$])$. Let $X$ be a toric Fano $d$-fold with $\rho_{X}=2 d-k$. If $d>3 k$, then $X$ is isomorphic to $\left(S_{3}\right)^{m} \times Y$, where $m$ is a positive integer and $Y$ is a toric Fano manifold of dimension at most $3 k$.

Note that necessarily here $\rho_{Y}=2 \operatorname{dim}(Y)-k$, since $\rho_{S_{3}}=4$. As will be explained below, this conjecture would be best possible. It would generalize the mentioned 
classification results, moreover, its validity would reduce the classification of toric Fano $d$-folds with $\rho_{X}=2 d-3$ to the case of $d \leq 9$, where complete classifications already exist.

The main result of this paper is the verification of the following weaker version of Conjecture 7.

Theorem 8. Let $X$ be a toric Fano d-fold with $\rho_{X}=2 d-k$. If $d>15 k^{2}+37 k+1$, then $X$ is isomorphic to $\left(S_{3}\right)^{m} \times Y$, where $m$ is a positive integer and $Y$ is a toric Fano manifold of dimension at most $15 k^{2}+37 k+1$.

This result is equivalent to Theorem 5 . Let us remark that it seems to be a hard open problem to improve the quadratic bound in Theorem 8 to a linear bound (as expected by Conjecture 7). See also Remark 40.

Remark 9. Casagrande's upper bound of $2 d$ on the Picard number was proven in more generality for $\mathbb{Q}$-factorial Gorenstein toric Fano varieties [7] (corresponding to simplicial reflexive polytopes). In [20] Øbro and the second author completely classified all such varieties with $\rho_{X}=2 d-1$. These results suggest that Conjecture 7 (and Theorem 8) should also hold when $X$ and $Y$ are $\mathbb{Q}$-factorial Gorenstein toric Fano varieties.

We say a toric Fano manifold $X$ is non- $S_{3}$-splittable, if it is not a product of $S_{3}$ and a lower-dimensional toric Fano manifold. Since there are only finitely many toric Fano $d$-folds of dimension $d \leq 15 k^{2}+37 k+1$, we get the following consequence from Theorem 8.

Corollary 10. For each $k$ there is only a finite number of isomorphism classes of non- $S_{3}$-splittable toric Fano $d$-folds $X$ with $\rho_{X} \geq 2 d-k$.

Let us illustrate the previous result by considering the number of isomorphism classes of non- $S_{3}$-splittable toric Fano manifolds for the known cases $k=0,1,2$. For $k=0$ we have just 1 such manifold (namely a point), for $k=1$ there are 3 (one in each dimension 1,2 , and 3 ), and for $k=2$ there are 15 isomorphism classes where the highest dimension is 6 (the numbers are 2,4,7,1,1 from dimension 2 up to dimension 6 respectively).

Regarding the sharpness of Conjecture 7, it is natural to reformulate and extend it in the following way (for an explicit description of the extreme case see Section 3).

Conjecture 11. Let $X$ be a non- $S_{3}$-splittable toric Fano $d$-fold. Then $\rho_{X} \leq \frac{5 d}{3}$, with equality if and only if $d=3 d^{\prime}$ (for $d^{\prime}$ a positive integer) and $X$ is isomorphic to the $d^{\prime}$-fold product of a uniquely determined toric $S_{3}$-bundle over $\mathbb{P}^{1}$.

Remark 12. In the non-toric setting much less is known about the structure of Fano manifolds with maximal Picard number. It is known that Fano 3-folds have Picard number at most 10 with equality only for the product of a Del Pezzo surface with $\mathbb{P}^{1}$. One conjecture in higher dimensions is that in even dimension $d$ the maximal Picard number equals $\frac{9 d}{2}$ and is attained only for products of del Pezzo surfaces with Picard number 9. There are further results [8, 9] that indicate that also in the non-toric setting extremizing the Picard number naturally leads to fibrations of Del Pezzo surfaces over another Fano manifold.

Let us finish this introduction by proposing the following further refinement of Casagrande's upper bound [7], based upon classification results in low dimensions. 
We say a toric Fano manifold $X$ is non-splittable if it is not a product of two lower-dimensional toric Fano manifolds.

Conjecture 13. Let $X$ be a non-splittable toric Fano $d$-fold, where $d \geq 3$. Then $\rho_{X} \leq \frac{4 d+3}{3}$, with equality if and only if $d=3 d^{\prime}$ (for $d^{\prime}$ a positive integer) and $X$ is isomorphic to a uniquely determined toric $\left(S_{3}\right)^{2 d^{\prime}}$-bundle over $\mathbb{P}^{d^{\prime}}$.

As mentioned above smooth Fano polytopes are already classified up to dimension 9. The conjectures are true for $d \leq 9$; see Section 3 .

1.2. Organization of the paper. This paper is organized as follows. Section 2 contains the combinatorial proof of Theorem 8. Section 3 discusses the equality cases in Conjecture 11 and Conjecture 13.

\section{PROOF OF THE MAIN THEOREM}

2.1. Preliminaries of the proof. Let $P$ be a $d$-dimensional smooth Fano polytope and $F$ a facet of $P$. Let us define some important notions and fix the notation (we refer to $[22,18])$.

For every vertex $v \in F$ there exists a unique neighboring facet $\mathrm{N}(F, v)$ that intersects with $F$ in a $(d-2)$-dimensional face that does not contain $v$. The unique vertex of $\mathrm{N}(F, v)$ that is not contained in $F$ is called the opposite vertex of $v$ with respect to $F$ and will be denoted by $\operatorname{opp}(F, v)$; see Figure 2 .

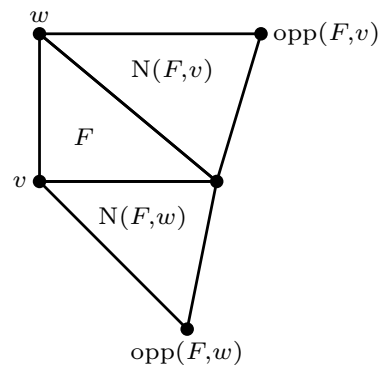

FiguRE 2. Neighboring facets and opposite vertices

We define the dual lattice of $N$ as $M:=\operatorname{Hom}_{\mathbb{Z}}(N, \mathbb{Z}) \cong \mathbb{Z}^{d}$ with the pairing $\langle\cdot, \cdot\rangle: M \times N \rightarrow \mathbb{Z}$. For Vert $F=\left\{v_{1}, \ldots, v_{d}\right\}$, a lattice basis of $N$, we get the dual lattice basis $u_{F, v_{1}}, \ldots, u_{F, v_{d}}$ of $M$. In other words, for $v \in \operatorname{Vert} F$ the lattice point $u_{F, v} \in M$ is characterized by $\left\langle u_{F, v}, v\right\rangle=1$ and $\left\langle u_{F, v}, w\right\rangle=0$ for $w \in \operatorname{Vert} F \backslash\{v\}$. Moreover, it holds that $u_{F}:=\sum_{i=1}^{d} u_{F, v_{i}} \in M$ is the unique primitive outer normal of $F$, i.e., for every $x \in \operatorname{Vert} F$ we have $\left\langle u_{F}, x\right\rangle=1$ and for every $y \in P$ we have $\left\langle u_{F}, y\right\rangle \leq 1$.

Let $j \in \mathbb{Z}$. We define the set of vertices of $P$ of level $j$ as

$$
V(F, j):=\left\{v \in \operatorname{Vert} P:\left\langle u_{F}, v\right\rangle=j\right\} .
$$

Moreover, we set $\eta_{j}^{F}:=|V(F, j)|$. Note that $\eta_{1}^{F}=d$ as $P$ is smooth and therefore simplicial. The vector $\eta^{F}:=\left(\eta_{1}^{F}, \eta_{0}^{F}, \eta_{-1}^{F}, \ldots\right)$ is called the $\eta^{F}$-vector of $P$.

An important role is played by the vertex sum $s_{P}$ of $P$ :

$$
s_{P}:=\sum_{v \in \operatorname{Vert} P} v
$$


We say that $F$ is a special facet [23], if $s_{P}$ is in the cone spanned by $F$. Note that in this case necessarily $\left\langle u_{F}, s_{P}\right\rangle \geq 0$. Special facets were the crucial tool in Øbro's classification algorithm for smooth Fano polytopes [21].

2.2. General assumptions of the proof. By a suitable lattice equivalence we can assume that we are from now on in the following situation:

$\triangleright P$ is a $d$-dimensional smooth Fano polytope,

$\triangleright \mid$ Vert $P \mid=3 d-k$ where $d \geq 3$ and $k \geq 3$ (for the case $k \leq 2$ see $[7,21,1]$ ),

$\triangleright F$ denotes a special facet which has outer normal vector $u_{F}$,

$\triangleright$ we write $\eta_{j}:=\eta_{j}^{F}$ for the number of vertices of $F$ on level $j$. We also use the notation $\eta_{\leq j}:=\sum_{i \leq j} \eta_{i}$.

2.3. Distribution of the vertices. Let us start by recalling some basic properties of vertices on level 0 :

Lemma 14 (Nill $[18$, Lem. 5.5]). Let $x \in V(F, 0)$. Then there exists a vertex $v \in \operatorname{Vert} F$ such that $x=\operatorname{opp}(F, v)$. Moreover, for $v \in \operatorname{Vert} F$ we have

$$
x=\operatorname{opp}(F, v) \quad \Longleftrightarrow\left\langle u_{F, v}, x\right\rangle<0 \quad \Longleftrightarrow \quad\left\langle u_{F, v}, x\right\rangle=-1 .
$$

Here the second equivalence follows from the smoothness of $P$ (for a more general statement see Lemma 18, where the opposite vertex is not necessarily assumed to be on level zero).

Lemma 15. The $\eta$-vector of any special facet of $P$ has the following properties:

$$
\begin{aligned}
\eta_{1} & =d \\
d-k \leq \eta_{0} & \leq d \\
d-2 k \leq \eta_{-1} & \leq d \\
\eta_{\leq-2} & \leq 2 k \\
\eta_{j} & =0 \text { for } j<-k-1 .
\end{aligned}
$$

Proof. The first equation is trivial, because $P$ is a smooth Fano polytope and therefore simplicial. The upper bound on $\eta_{0}$ follows from Lemma 14, since there are at most $d$ opposite vertices to the $d$ vertices in a given facet. The upper bound for $\eta_{-1}$ comes from the fact that we are looking at a special facet $F$ and therefore we have

$$
0 \leq\left\langle u_{F}, s_{P}\right\rangle=\sum_{i \leq 1} i \cdot \eta_{i}=d+\sum_{i \leq-1} i \cdot \eta_{i}
$$

Now the upper bound follows from:

$$
d \geq \sum_{i \leq-1}|i| \cdot \eta_{i} \geq \sum_{i \leq-1} \eta_{i}
$$

With this inequality we also get the lower bound on $\eta_{0}$ since $\mid$ Vert $P \mid=\sum_{i \leq 1} \eta_{i}$ gives us

$$
3 d-k=d+\eta_{0}+\sum_{i \leq-1} \eta_{i} \leq d+\eta_{0}+d .
$$

Solving this for $\eta_{0}$ we get $d-k \leq \eta_{0}$. 
The lower bound for $\eta_{-1}$ follows with a similar argument. From the equality $\mid$ Vert $P \mid=\sum_{i \leq 1} \eta_{i}$ we get

$$
3 d-k-\eta_{1}-\eta_{0}-\eta_{-1}=\sum_{i \leq-2} \eta_{i}
$$

Using the fact that we have a special facet and using the upper bounds on $\eta_{0}$ and $\eta_{1}$ gives us

$$
\begin{aligned}
0 & \leq\left\langle u_{F}, s_{P}\right\rangle \\
& =\sum_{i \leq 1} i \cdot \eta_{i} \\
& =d-\eta_{-1}+\sum_{i \leq-2} i \cdot \eta_{i} \\
& \leq d-\eta_{-1}-2 \sum_{i \leq-2} \eta_{i} \\
& =d-\eta_{-1}-2\left(3 d-k-\eta_{1}-\eta_{0}-\eta_{-1}\right) \\
& \leq d-\eta_{-1}-6 d+2 k+2 d+2 d+2 \eta_{-1} \\
& =\eta_{-1}-d+2 k .
\end{aligned}
$$

Now, the upper bound for $\eta_{\leq-2}$ is a direct consequence of the previous lower bounds:

$$
\begin{aligned}
\sum_{i \leq-2} \eta_{i} & =3 d-k-\eta_{1}-\eta_{0}-\eta_{-1} \\
& \leq 3 d-k-d-(d-k)-(d-2 k)=2 k
\end{aligned}
$$

Finally, let $j<0$ be the minimal level with $\eta_{j} \neq 0$. Let $v \in V(F, j)$. Then there are at least $3 d-k-\eta_{1}-\eta_{0}-1=2 d-k-1-\eta_{0}$ vertices on negative levels apart from $v$. In particular,

$$
\begin{aligned}
0 & \leq\left\langle u_{F}, s_{P}\right\rangle \\
& \leq d-\left(2 d-k-1-\eta_{0}\right)+j \\
& =-d+k+1+\eta_{0}+j,
\end{aligned}
$$

hence $j \geq d-\eta_{0}-k-1 \geq-k-1$, since $\eta_{0} \leq d$.

Lemma 16. The level of $s_{P}$ is at most $k$.

Proof. The maximal level of $s_{P}$ is achieved by having $d$ vertices on level $1, d$ vertices on level 0 , and the remaining vertices ( $d-k$ many) on level -1 .

2.4. Partitioning the vertex set of $F$. We will use the following notation introduced in [1].

Definition 17. We call a vertex $v$ of the facet $F \operatorname{good}$ if $\operatorname{opp}(F, v)$ is contained in $V(F, 0)$.

As the following well-known result shows, the second condition of a good vertex given in [1] is automatically true in our setting because we assume smoothness.

Lemma 18. If $v$ is a vertex of $F$, then $\left\langle u_{F, v}, \operatorname{opp}(F, v)\right\rangle=-1$. 


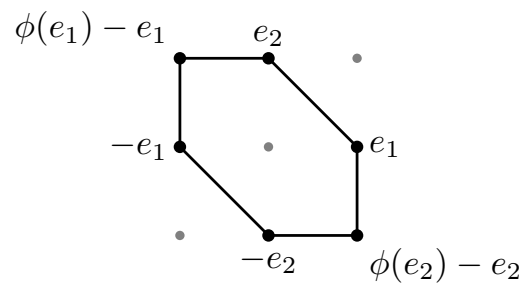

Figure 3 . The hexagon $P_{6}$ with labeled vertices.

Let us also recall the notation of the $\phi$-function (of a facet $F$ ) used in [1]:

$$
\phi: \operatorname{Vert} F \rightarrow \operatorname{Vert} F \cup\{0\}, v \mapsto \begin{cases}w & \text { if } w:=\operatorname{opp}(F, v)+v \in \operatorname{Vert} F \\ 0 & \text { otherwise } .\end{cases}
$$

Note that if $\phi(v) \neq 0$, then $v$ is a good vertex of $F$, but there are good vertices for which $\phi(v)=0$, so the converse is not true.

Definition 19. We will partition the set Vert $F$ of size $d$ into three different groups:

$\triangleright A:=\{u \in \operatorname{Vert} F: u$ is good, $\phi(u) \neq 0\}$,

$\triangleright B:=\{v \in \operatorname{Vert} F: v$ is good, $\phi(v)=0\}$,

$\triangleright C:=\{w \in \operatorname{Vert} F: w$ is not good $\}$.

The following result clarifies the geometric meaning of a good vertex being in $A$ or $B$.

Lemma 20. Let $v$ be a good vertex of the facet $F$ (i.e., $v \in A \cup B$ ). Then $\operatorname{opp}(F, w) \neq \operatorname{opp}(F, v)$ for every vertex $w$ of $F$ other than $v$ if and only if $\phi(v) \neq 0$ (i.e., $v \in A$ ).

Proof. The 'only if' part is proven in [1, Lemma 25]. For the converse assume that $\phi(v) \neq 0$ and $x:=\operatorname{opp}(F, w)=\operatorname{opp}(F, v)$ for some vertex $w \neq v$ of $F$. By the second condition, $x$ and $v$ lie in the neighboring facet $\mathrm{N}(F, w)$. Hence, $x+v$ cannot be a vertex of $P$, a contradiction to $\phi(v) \neq 0$.

Example 21. In Figure 3 the behaviour of the $\phi$-function is illustrated. The vertices $e_{1}$ and $e_{2}$ are both good and belong to the partition set $A$. We have $\phi\left(e_{1}\right)=e_{2}$ and $\phi\left(e_{2}\right)=e_{1}$.

Let us deduce the following observations on the sizes of the sets $A, B, C$.

\section{Lemma 22.}

$$
d-2 k \leq|A|, \quad|B|+|C| \leq 2 k, \quad|C| \leq k .
$$

Proof. By Lemma 14 every vertex on level 0 must be opposite to some vertex in $F$, and clearly two different vertices on level 0 cannot be opposite to the same vertex in $F$, as opposite vertices are unique. Therefore, the bound $\eta_{0} \geq d-k$, see Lemma 15 , implies that at least $d-k$ vertices have pairwise distinct opposite vertices on level 0 . In particular, at least $d-k$ vertices of $F$ must be good, i.e., $|C| \leq k$. Moreover, there can be at most $k$ of these $d-k$ good vertices, say $v$, that have the property that there exists another vertex $\bar{v} \in \operatorname{Vert} F$ with $\operatorname{opp}(F, \bar{v})=\operatorname{opp}(F, v)$. Therefore, Lemma 20 implies $|A| \geq d-2 k$, and thus $|B|+|C| \leq 2 k$. 
Example 23. The reader should be aware that we do not claim that $|B| \leq k$. In fact, $|B|=2 k$ is possible as the following example shows. For this, consider the convex hull of the following 10 points in dimension $d=4$ :

$$
\begin{gathered}
e_{1}, e_{2}, e_{3}, e_{4} \\
-e_{1}-e_{2}+e_{3}+e_{4},-e_{3}-e_{4}+e_{1}+e_{2} \\
-e_{1},-e_{2},-e_{3},-e_{4} .
\end{gathered}
$$

This polytope is indeed a smooth Fano polytope with 10 vertices, so $k=2$, as $3 \cdot d=12$. The vertices $e_{1}, e_{2}, e_{3}, e_{4}$ form a special facet $F$. Here, all the vertices of $F$ are in the partition set $B$, e.g., $e_{1}$ and $e_{2}$ share the same opposite vertex $-e_{1}-e_{2}+e_{3}+e_{4}$. Therefore $|B|=4=2 k$. This example can be generalized to higher dimensions, where all the vertices of a special facet $F$ are good, and yet $k$ pairs of vertices share an opposite vertex.

Let us summarize what we know about the opposite vertices (keep in mind that Vert $F$ forms a lattice basis).

Proposition 24. Let $z \in \operatorname{Vert} F$.

(i) If $z \in A$, then $\operatorname{opp}(F, z)=-z+\phi(z)$.

(ii) If $z \in B$, then

$$
\operatorname{opp}(F, z)=-z+\sum_{u \in A} a_{u} u+\sum_{v \in B \backslash\{z\}} b_{v} v+\sum_{w \in C} c_{w} w
$$

with $a_{u}, c_{w} \in \mathbb{Z}_{\geq 0}$ and $b_{v} \in \mathbb{Z}_{\geq-1}$ for all $u \in A, v \in B \backslash\{z\}$ and $w \in C$. Further it holds

$$
\sum_{u \in A} a_{u}+\sum_{v \in B \backslash\{z\}} b_{v}+\sum_{w \in C} c_{w}=1, \quad \sum_{u \in A} a_{u} \leq k+1, \quad \sum_{v \in B \backslash\{z\}} b_{v} \geq-k .
$$

(iii) If $z \in C$, then

$$
\operatorname{opp}(F, z)=-z+\sum_{u \in A} a_{u} u+\sum_{v \in B} b_{v} v+\sum_{w \in C \backslash\{z\}} c_{w} w
$$

with $a_{u}, b_{v}, c_{w} \in \mathbb{Z}$ for all $u \in A, v \in B$ and $w \in C \backslash\{z\}$. Further it holds

$$
\sum_{u \in A} a_{u}+\sum_{v \in B} b_{v}+\sum_{w \in C \backslash\{z\}} c_{w} \leq 0 .
$$

Proof. (i) Follows by definition.

(ii) The first statement is a consequence of Lemma 14 and Lemma 20. The second equation follows since $\operatorname{opp}(F, v)$ is on level 0 . Finally, as in the proof of Lemma 22, we note that for $z \in B$, there can be at most $k$ other vertices $\bar{z} \in \operatorname{Vert} F$ (necessarily, $\bar{z} \in B$ ) with $\operatorname{opp}(F, \bar{z})=\operatorname{opp}(F, z)$. By Lemma 14 this implies that there are at most $k$ other vertices $\bar{z} \in B, \bar{z} \neq z$, with $b_{\bar{z}}=\left\langle u_{F, \bar{z}}, \operatorname{opp}(F, z)\right\rangle<0$ (equivalently, equal to -1 ). We deduce that $\sum_{v \in B \backslash\{z\}} b_{v} \geq-k$. Since $\operatorname{opp}(F, z) \in V(F, 0)$, this implies also that $\sum_{v \in A} a_{u} \leq k+1$.

(iii) This is as before a direct consequence of Lemma 18 together with the fact that $z$ is not a good vertex and so the opposite vertex must lie on a level below 0 . 
2.5. Vertices on negative levels. Let us recall some of Øbro's observations.

Lemma 25 (Øbro [23, Lem. 1 and 2]). Let $G$ be a facet of $P, z$ a vertex of $G$, and $G^{\prime}:=\mathrm{N}(G, z)$. Then $u_{G^{\prime}}=u_{G}+\left(\left\langle u_{G^{\prime}}, z\right\rangle-1\right) u_{G, z}$. Moreover, for $x \in P$ we have $\left\langle u_{G}, x\right\rangle-1 \leq\left\langle u_{G, z}, x\right\rangle$. In case of equality we have $x=\operatorname{opp}(G, z)$.

Corollary 26. If $z \in A \cup B$, then $u_{F^{\prime}}=u_{F}-u_{F, z}$ for $F^{\prime}:=\mathrm{N}(F, z)$.

Proof. This follows from plugging in $x:=\operatorname{opp}(F, z)$ into Lemma 25.

The following observation is contained in Øbro's PhD thesis [22, Lemma 1.9(5)].

Lemma 27. Let $x$ be a vertex of $P$ with $x \neq \operatorname{opp}(F, z)$ for some vertex $z \in \operatorname{Vert} F$. Then the inequality $\left\langle u_{F, z}, x\right\rangle<0$ implies $\left\langle u_{F}, x\right\rangle\left\langle\left\langle u_{F}, \operatorname{opp}(F, z)\right\rangle\right.$. In other words, if $z$ has a negative contribution to $x$ then the vertex $x$ must lie on a level strictly lower than the opposite vertex of $z$.

As we know that for all $w \in C$ the vertex $\operatorname{opp}(F, w)$ lies strictly below level 0 , we get the following consequence.

Corollary 28. There is at most one vertex $x \in V(F,-1)$ with $\left\langle u_{F, w}, x\right\rangle<0$ for some $w \in C$. If such a vertex $x$ exists, then $x=\operatorname{opp}(F, w)$.

Let us make some auxiliary considerations regarding vertices on negative levels.

Lemma 29. Let $z \in A \cup B$. For every $\bar{z} \in \operatorname{Vert} F$ there is at most one vertex $x \in V(F,-1)$ with $\left\langle u_{F, z}, x\right\rangle=-1$ and $\left\langle u_{F, \bar{z}}, x\right\rangle<\left\langle u_{F, \bar{z}}, \operatorname{opp}(F, z)\right\rangle$.

Proof. Let us denote $y=\operatorname{opp}(F, z)$. We have $\left\langle u_{F}, y\right\rangle=0$, since $z$ is good. Suppose there are two vertices $x, \bar{x} \in V(F,-1)$ with $\left\langle u_{F, z}, x\right\rangle=\left\langle u_{F, z}, \bar{x}\right\rangle=-1$ and $\left\langle u_{F, \bar{z}}, x\right\rangle<$ $\left\langle u_{F, \bar{z}}, y\right\rangle$ as well as $\left\langle u_{F, \bar{z}}, \bar{x}\right\rangle\left\langle\left\langle u_{F, \bar{z}}, y\right\rangle\right.$ for some $\bar{z} \in \operatorname{Vert} F$. We rewrite $x, \bar{x}$ and $y$ as:

$$
x=\sum_{v \in \operatorname{Vert} F} \overbrace{\left\langle u_{F, v}, x\right\rangle}^{\lambda_{v}:=} v, \quad \bar{x}=\sum_{v \in \operatorname{Vert} F} \overbrace{\left\langle u_{F, v}, \bar{x}\right\rangle}^{\mu_{v}:=} v, \quad y=\sum_{v \in \operatorname{Vert} F} \overbrace{\left\langle u_{F, v}, y\right\rangle}^{\alpha_{v}:=} v .
$$

Considering the neighboring facet $F^{\prime}:=\mathrm{N}(F, z)$ and its outer facet normal $u_{F^{\prime}}$, Corollary 26 implies $u_{F^{\prime}}=u_{F}-u_{F, z}$, so $x$ and $\bar{x}$ both lie on level 0 with respect to $F^{\prime}$. We rewrite $x$ and $\bar{x}$ in terms of the new lattice basis Vert $F^{\prime}=\operatorname{Vert} F \backslash\{z\} \cup\{y\}$ :

$$
x=y+\sum_{v \neq z} \underbrace{\left(\lambda_{v}-\alpha_{v}\right)}_{=\left\langle u_{F^{\prime}, v}, x\right\rangle} v, \quad \bar{x}=y+\sum_{v \neq z} \underbrace{\left(\mu_{v}-\alpha_{v}\right)}_{=\left\langle u_{F^{\prime}, v}, \bar{x}\right\rangle} v,
$$

where we used $\lambda_{z}=\mu_{z}=\alpha_{z}=-1$. Our assumptions on $\bar{z}$ gives us that $\lambda_{\bar{z}}<\alpha_{\bar{z}}$ and $\mu_{\bar{z}}<\alpha_{\bar{z}}$. So Lemma 14 yields $x=\operatorname{opp}\left(F^{\prime}, \bar{z}\right)=\bar{x}$.

Lemma 30. Let $x, \bar{x} \in \operatorname{Vert} P$ with $\left\langle u_{F}, x\right\rangle\left\langle\left\langle u_{F}, \bar{x}\right\rangle\right.$. Then there must exist at least one vertex $z \in \operatorname{Vert} F$ with $\left\langle u_{F, z}, x\right\rangle<\left\langle u_{F, z}, \bar{x}\right\rangle$.

Proof. Suppose this is not true, so $\left\langle u_{F, z}, x\right\rangle \geq\left\langle u_{F, z}, \bar{x}\right\rangle$ for every $z \in \operatorname{Vert} F$. Then we get a contradiction from

$$
\left\langle u_{F}, x\right\rangle=\sum_{z \in \operatorname{Vert} F}\left\langle u_{F, z}, x\right\rangle \geq \sum_{z \in \operatorname{Vert} F}\left\langle u_{F, z}, \bar{x}\right\rangle=\left\langle u_{F}, \bar{x}\right\rangle .
$$


Lemma 31 (Øbro [23, Lem. 5]). Let $v$ and $w \neq v$ be good vertices of the facet $F$ with $\operatorname{opp}(F, v) \neq \operatorname{opp}(F, w)$. Then there exists no vertex $x \in V(F,-1)$ such that $\left\langle u_{F, v}, x\right\rangle=\left\langle u_{F, w}, x\right\rangle=-1$.

Now we can give a rough classification of vertices in $V(F,-1)$.

Proposition 32. Any vertex $x \in V(F,-1)$ is one of the following three types:

(i) $x=\operatorname{opp}(F, z)$ for some $z \in C$ with $\left\langle u_{F, z}, x\right\rangle<0$.

(ii) $\left\langle u_{F, z}, x\right\rangle=-1$ for some $z \in B,\left\langle u_{F, v}, x\right\rangle \geq-1$ for all $v \in B$, and $\left\langle u_{F, w}, x\right\rangle \geq 0$ for all $w \in A \cup C$.

(iii) $x=-z$ for some $z \in A$.

There are at most $|C|$ vertices of type (i), at most $(k+1)|B|$ vertices of type (ii), and at least $\eta_{-1}-|C|-|B|(k+1)$ vertices of type (iii).

Proof. We consider the following situation: $x \in V(F,-1)$ and $z \in \operatorname{Vert} F$ with $\left\langle u_{F, z}, x\right\rangle<0$ (clearly, such a $z$ must exist). The proof proceeds in three steps.

First, suppose $z \in C$. Corollary 28 implies that $x$ is the only vertex on level -1 which satisfies $\left\langle u_{F, z}, x\right\rangle<0$ (namely, the opposite vertex to $z$ ). Hence, $x$ is of type (i).

Secondly, let us look at the remaining vertices $x$ on level -1 (of which there are at least $\eta_{-1}-|C|$ many), i.e., $\left\langle u_{F, \bar{z}}, x\right\rangle \geq 0$ for any $\bar{z} \in C$. Suppose we have $z \in B$ with $\left\langle u_{F, z}, x\right\rangle\left\langle 0\right.$. Lemma 25 implies $\left\langle u_{F, z}, x\right\rangle=-1$. We define $y:=\operatorname{opp}(F, z) \in V(F, 0)$. We claim that $\left\langle u_{F, \bar{z}}, x\right\rangle \geq 0$ for all $\bar{z} \in \operatorname{Vert} F$ which satisfy $\operatorname{opp}(F, \bar{z}) \neq y$. Clearly, this is automatically true for $\bar{z} \in C$ by our choice of $x$. Otherwise, $\bar{z}$ is good, and the claim follows from Lemma 31. Hence, we get (use Lemmas 14, 18 and 25 again):

$$
\begin{aligned}
& \bar{z} \in \operatorname{Vert} F \text { with } \operatorname{opp}(F, \bar{z}) \neq y \Longrightarrow\left\langle u_{F, \bar{z}}, y\right\rangle \geq 0 \text { and }\left\langle u_{F, \bar{z}}, x\right\rangle \geq 0 \\
& \bar{z} \in \operatorname{Vert} F \text { with } \operatorname{opp}(F, \bar{z})=y \Longrightarrow\left\langle u_{F, \bar{z}}, y\right\rangle=-1 \text { and }\left\langle u_{F, \bar{z}}, x\right\rangle \geq-1
\end{aligned}
$$

Here we see that $\left\langle u_{F, \bar{z}}, x\right\rangle=-1$ is only possible if $\bar{z} \in B$ with $\operatorname{opp}(F, \bar{z})=y$ (see also Proposition 24(ii)). From these observations we deduce that $x$ is of type (ii). Now, Lemma 30 implies that there must exist at least one vertex $\bar{z} \in \operatorname{Vert} F$ with $\left\langle u_{F, \bar{z}}, x\right\rangle\left\langle\left\langle u_{F, \bar{z}}, y\right\rangle\right.$ because $x$ lies on level -1 and $y$ is on level 0. From (2) we observe that this implies $\left\langle u_{F, \bar{z}}, y\right\rangle>0$. By Proposition 24(ii), $y$ can have at most $(k+1)$ many negative coordinates and lies on level 0 so we see that $\left\langle u_{F, \bar{z}}, y\right\rangle>0$ is only possible for at most $(k+1)$ choices for $\bar{z} \in \operatorname{Vert} F$. But Lemma 29 tells us that $x$ is uniquely determined by $x \in V(F,-1),\left\langle u_{F, z}, x\right\rangle=-1$ and $\left\langle u_{F, \bar{z}}, x\right\rangle\left\langle\left\langle u_{F, \bar{z}}, y\right\rangle\right.$ for given $\bar{z} \in \operatorname{Vert} P$. Therefore, for given vertex $z \in B$, there are at most $(k+1)$ vertices $x \in V(F,-1)$ that are not opposite to a vertex in $C$ and satisfy $\left\langle u_{F, z}, x\right\rangle<0$.

Finally, consider the remaining vertices $x$ on level -1 , of which there are at least $\eta_{-1}-|C|-|B|(k+1)$ many, i.e., $\left\langle u_{F, \bar{z}}, x\right\rangle \geq 0$ for all $\bar{z} \in B \cup C$. In this case, we must have $z \in A$ with $\left\langle u_{F, z}, x\right\rangle<0$ for such an $x$. Again, Lemma 25 shows that this is equivalent to $\left\langle u_{F, z}, x\right\rangle=-1$. Now, Lemma 31 implies $\left\langle u_{F, \bar{z}}, x\right\rangle \geq 0$ for all $\bar{z} \in A \backslash\{z\}$. Since $\sum_{v \in \operatorname{Vert} F}\left\langle u_{F, v}, x\right\rangle v=x$ with $\sum_{v \in \operatorname{Vert} F}\left\langle u_{F, v}, x\right\rangle=-1$ and there is only one negative summand which is already -1 , we must have $x=-z$, so $x$ is of type (iii).

2.6. Construction of the splitting. We will now restrict our set $A$.

Definition 33. Let

$$
A^{\prime}:=\{v \in A:-v \in \operatorname{Vert} P\} .
$$


Lemma 34 (Assarf, Joswig, Paffenholz [1, Lemma 29]). If $v \in A^{\prime}$, in particular $\phi(v) \neq 0$, then $\phi(\phi(v)) \in\{0, v\}$.

We want to restrict $A^{\prime}$ further to a subset of elements $v$ that have the desirable property $\phi(\phi(v))=v$. For this, let us first recall the following result.

Lemma 35 (Casagrande [6, Lemma 3.3]). If $z, x, x^{\prime}, y, y^{\prime} \in \operatorname{Vert} P$ with $x \neq x^{\prime}$ such that $x+z=y$ and $x^{\prime}+z=y^{\prime}$, then $y^{\prime}=-x$.

Corollary 36. Let $z \in \operatorname{Vert} F$. Then

$$
\left|\left\{v \in A^{\prime} \mid \phi(v)=z\right\}\right| \leq 1 .
$$

Proof. Assume there exist $v, v^{\prime} \in A^{\prime}, v \neq v^{\prime}$ with $\phi(v)=z=\phi\left(v^{\prime}\right)$. We define

$$
x:=-v, \quad x^{\prime}:=-v^{\prime}, \quad y:=\operatorname{opp}(F, v)=z-v, \quad y^{\prime}:=\operatorname{opp}\left(F, v^{\prime}\right)=\phi\left(v^{\prime}\right)-v^{\prime}
$$

Then Lemma 35 implies $\phi\left(v^{\prime}\right)-v^{\prime}=y^{\prime}=-x=v$, a contradiction since $\phi\left(v^{\prime}\right)-v^{\prime}$ is on level 0 , while $v$ is on level 1 .

Next, let us write the vertex sum as a linear combination of vertices of $F$ :

$$
s_{P}=\sum_{z \in \operatorname{Vert} F} \gamma_{z} z
$$

Note that $\gamma_{z} \geq 0$ for any $z \in \operatorname{Vert} F$. Now, we can restrict the set of good vertices further.

Definition 37. Let

$$
\bar{A}:=\left\{v \in A^{\prime}: \phi(v) \in A^{\prime}, \gamma_{v}=0=\gamma_{\phi(v)}\right\} .
$$

Note that Lemma 34 implies $\phi(\phi(v))=v$ for $v \in \bar{A}$. In particular, $v \in \bar{A}$ implies $\phi(v) \in \bar{A}$.

The reason for including the condition on the vertex sum in the definition of $\bar{A}$ is that changing the special facet will be useful in the upcoming proofs, and this condition will ensure that the new facet will still be special.

Note that $\bar{A}$ splits into disjoint pairs, each pair, say $v, \phi(v)$, giving rise to the following configuration of vertices of $P$ (see also Example 21 and Figure 3):

$$
\begin{gathered}
v, \phi(v) \\
v-\phi(v), \phi(v)-v \\
-\phi(v),-v
\end{gathered}
$$

We can now estimate the size of the set $\bar{A}$.

\section{Proposition 38.}

$$
|\bar{A}| \geq 2\left|A^{\prime}\right|-d-2 k .
$$

Proof. We have by Corollary 36

$\left|\left\{v \in A^{\prime}: \phi(v) \in B \cup C\right\} \cup\left\{v \in A^{\prime}: \phi(v) \in A \backslash A^{\prime}\right\}\right| \leq|B|+|C|+|A|-\left|A^{\prime}\right|$.

This shows that

$$
\left\{v \in A^{\prime}: \phi(v) \in A^{\prime}\right\} \geq\left|A^{\prime}\right|-\left(|B|+|C|+|A|-\left|A^{\prime}\right|\right)=2\left|A^{\prime}\right|-d .
$$

Finally, from Lemma 16 we see that there are at most $k$ vertices in $F$ with nonzero $\gamma$-coordinate. Hence, we have to potentially remove $k$ pairs, so $2 k$ vertices from the previous estimate. 
In order to finally prove that we indeed have a splitting into a sufficiently large subset of these hexagons it is necessary to bound the number of its nonzero coordinates in $\bar{A}$ for each vertex on a negative level.

Lemma 39. For $x \in V(F, \leq-1)$ it holds

$$
\left|\left\{z \in \bar{A} \mid\left\langle u_{F, z}, x\right\rangle \neq 0\right\}\right| \leq 2 k+2 .
$$

Proof. Let $x$ be a vertex in $V(F, \leq-1)$. Suppose there exists a vertex $v_{0} \in \bar{A}$ with $\left\langle u_{F, v_{0}}, x\right\rangle<0$, then we consider the neighboring facet $F_{1}:=\mathrm{N}\left(F, v_{0}\right)$. Note that by our assumption on $\bar{A}$, the facet $F_{1}$ is still a special facet, as $F_{1}$ has vertices Vert $F \backslash\left\{v_{0}\right\} \cup\left\{\phi\left(v_{0}\right)-v_{0}\right\}$. Hence, $u_{F_{1}}=u_{F}-u_{F, v_{0}}$. In particular, we get $\left\langle u_{F_{1}}, x\right\rangle>\left\langle u_{F}, x\right\rangle$.

Now, suppose there exists another vertex $v_{1} \in \bar{A}$ with $v_{1} \notin\left\{v_{0}, \phi\left(v_{0}\right)\right\}$ such that $\left\langle u_{F, v_{1}}, x\right\rangle<0$. Note that by the choice of $v_{1}$ and by Lemma 25 and the fact that $u_{F_{1}, v_{1}}=u_{F, v_{1}}$, the opposite vertex of $v_{1}$ with respect to $F_{1}$ is the same as for $F$, that is $\operatorname{opp}\left(F_{1}, v_{1}\right)=\operatorname{opp}\left(F, v_{1}\right)=\phi\left(v_{1}\right)-v_{1}$. Consider $F_{2}:=\mathrm{N}\left(F_{1}, v_{1}\right)$. Again, by Lemma 14, the facet $F_{2}$ has vertices

$$
\text { Vert } F \backslash\left\{v_{0}, v_{1}\right\} \cup\left\{\phi\left(v_{0}\right)-v_{0}\right\} \cup\left\{\phi\left(v_{1}\right)-v_{1}\right\}
$$

and therefore is also a special facet. Hence, $u_{F_{2}}=u_{F}-u_{F, v_{0}}-u_{F, v_{1}}$. In particular, $\left\langle u_{F_{2}}, x\right\rangle>\left\langle u_{F_{1}}, x\right\rangle$.

Continuing with this argumentation we might end up with a facet $F_{\ell}$ where $\left\langle u_{F_{\ell}}, x\right\rangle=0$ and vertices

$$
\text { Vert } F \backslash\left\{v_{0}, \ldots, v_{\ell}\right\} \cup\left\{\phi\left(v_{0}\right)-v_{0}\right\} \cup \cdots \cup\left\{\phi\left(v_{\ell-1}\right)-v_{\ell-1}\right\} \text {. }
$$

At this moment there cannot exist another vertex $v_{\ell} \in \bar{A}$ with $\left\langle u_{F, v_{\ell}}, x\right\rangle<0$, since Lemma 14 shows that $x=\operatorname{opp}\left(F_{\ell}, v_{\ell}\right)=\phi\left(v_{\ell}\right)-v_{\ell} \in V(F, 0)$ (note that $u_{F_{\ell}, v_{\ell}}=u_{F, v_{\ell}}$ and due to our choices the opposite vertices of Vert $F_{\ell} \cap \bar{A}$ do not change). A contradiction.

So the number of vertices $v$ with $\left\langle u_{F, v}, x\right\rangle<0$ is at most $2 \cdot\left|\left\langle u_{F}, x\right\rangle\right|$, where the factor 2 comes from the fact that $\left\langle u_{F, \phi\left(v_{i}\right)}, x\right\rangle$ could be zero or not.

One can repeat the same argument with $\left\langle u_{F, v}, x\right\rangle>0$. In this case, since all the facets were special, we can use that $-k-1$ is the lowest possible level (Lemma 15) to deduce that there are at most $2 \cdot\left(k+1-\left|\left\langle u_{F}, x\right\rangle\right|\right)$ vertices $v \in \bar{A}$ with $\left\langle u_{F, v}, x\right\rangle>0$. Putting this together we get the desired statement.

\subsection{The proof of Theorem 5. Let us define}

$$
W:=\bigcup_{v \in \bar{A}}\{v, \phi(v), \phi(v)-v, v-\phi(v),-v,-\phi(v)\} .
$$

Note that the convex hull of these vertices splits into hexagons as desired. Our goal is now to bound the number of vertices $v \in \bar{A}$ for which there exists some vertex $x \in \operatorname{Vert} P \backslash W$ with $\left\langle u_{F, v}, x\right\rangle \neq 0$. Then after removing these undesired vertices $v$ (and their hexagons) from $W$, the remaining vertices of $W$ live in a subspace that is transversal to all other vertices of $P$.

First let us consider the case that $x \in V(F, 0) \backslash W$ with $\left\langle u_{F, v}, x\right\rangle \neq 0$ for some $v \in \bar{A}$. In particular, $x$ is not opposite to any vertex in $\bar{A}$ (since $x \notin W$ ). By Lemma 14, this implies $\left\langle u_{F, v}, x\right\rangle>0$, and $x=\operatorname{opp}\left(F, v^{\prime}\right)$ for some $v^{\prime} \in \operatorname{Vert} F$ with $v^{\prime} \in(A \backslash \bar{A}) \cup B$. We distinguish two cases. If $v^{\prime} \in A \backslash \bar{A}$, then Proposition 24 implies $\phi\left(v^{\prime}\right)=v$ and $x=v-v^{\prime}$. (Since in this case each choice of $v^{\prime}$ uniquely determines $x$ 
and thus $v$, there are at most $|A \backslash \bar{A}|$ many possibilities for $v$.) We can say more for $v^{\prime} \in B$. Let us partition $B$ into disjoint subsets $B_{1}, \ldots, B_{t}$, where two vertices of $B$ are in the same subset $B_{i}$ (for $i \in\{1, \ldots, t\}$ ) if and only if their opposite vertices are the same. Let us assume that $v^{\prime} \in B_{j}$ for some $j \in\{1, \ldots, t\}$. By Lemma 14 and Proposition 24, the vertex $x$ has negative coordinates only in $B_{j}$, so $x$ has at most $\left|B_{j}\right|$ many positive coordinates in $\bar{A}$, since $x$ is on level 0 . So, in this second case there are at most $t$ possibilities for $x$, and at most $\left|B_{1}\right|+\cdots+\left|B_{t}\right|=|B|$ many possibilities for $v$. Hence, the number of vertices $v \in \bar{A}$ for which there exists some vertex $x \in V(F, 0) \backslash W$ with $\left\langle u_{F, v}, x\right\rangle \neq 0$ is bounded by

$$
|A|-|\bar{A}|+|B|
$$

Next we notice that there are at most $2 k$ vertices in $V(F, \leq-2)$ by Lemma 15 . For each such vertex $x$, Lemma 39 implies that $\left\langle u_{F, v}, x\right\rangle \neq 0$ can be satisfied for at most $2 k+2$ many choices of $v \in \bar{A}$. Therefore, the number of vertices $v \in \bar{A}$ for which there exists some vertex $x \in V(F, \leq-2) \backslash W$ with $\left\langle u_{F, v}, x\right\rangle \neq 0$ is bounded by

$$
(2 k+2) 2 k \text {. }
$$

This leaves the case where $x \in V(F,-1)$ with $-x \notin \bar{A}$ and $\left\langle u_{F, v}, x\right\rangle \neq 0$ for some $v \in \bar{A}$. By Proposition 32 the vertex $x$ must be of type (i) or (ii). Lemma 39 shows that the number of vertices $v \in \bar{A}$ for which there exists some vertex $x \in V(F,-1) \backslash W$ of type (i) with $\left\langle u_{F, v}, x\right\rangle \neq 0$ is bounded by

$$
(2 k+2)|C| \text {. }
$$

Let us define two sets. $\tilde{V}$ is the set of vertices in $V(F,-1)$ of type (ii), and $\tilde{A}$ is the set of vertices $v \in \bar{A}$ where there exists some $x \in \tilde{V}$ with $\left\langle u_{F, v}, x\right\rangle \neq 0$ (necessarily, $>0$ ). Note that by the description of a vertex $x \in \tilde{V}$ in Proposition 32 it holds that, since $x \in V(F,-1)$, if $j$ many coordinates of $x$ in $\bar{A}$ are positive, then at least $j+1$ many coordinates of $x$ in $B$ must be equal to -1 . Hence,

$$
\begin{aligned}
|\tilde{A}|+|\tilde{V}| & \leq \sum_{x \in \tilde{V}}\left(\left|\left\{v \in \tilde{A}:\left\langle u_{F, v}, x\right\rangle>0\right\}\right|+1\right) \\
& \leq \sum_{x \in \tilde{V}}\left|\left\{v \in B:\left\langle u_{F, v}, x\right\rangle=-1\right\}\right| \\
& =\sum_{v \in B}\left|\left\{x \in \tilde{V}:\left\langle u_{F, v}, x\right\rangle=-1\right\}\right| \\
& \leq(k+1)|B|,
\end{aligned}
$$

where we used Proposition 32 for the last inequality. This shows that the number of vertices $v \in \bar{A}$ for which there exists some vertex $x \in V(F,-1) \backslash W$ of type (ii) with $\left\langle u_{F, v}, x\right\rangle \neq 0$ is bounded by

$$
(k+1)|B|-\tilde{V}
$$

Summing up Equations (3),(4),(5), and (6) we get that the number of vertices $v \in \bar{A}$ for which there exists some vertex $x \in \operatorname{Vert} P \backslash W$ with $\left\langle u_{F, v}, x\right\rangle \neq 0$ is bounded by

$$
|A|-|\bar{A}|+|B|+(2 k+2) 2 k+(2 k+2)|C|+(k+1)|B|-|\tilde{V}| .
$$


Using $|A|+|B|+|C|=d$ and $|\tilde{V}| \geq \eta_{-1}-|C|-\left|A^{\prime}\right|$ (see Proposition 32), this expression can be bounded from above by

$$
(2 k+2) 2 k+(2 k+2)|C|+(k+1)|B|+\left|A^{\prime}\right|-|\bar{A}|-\eta_{-1}+d .
$$

Now, we want to remove all these vertices $v$ together with $\phi(v)$. So, we deduce that the number of vertices $v \in \bar{A}$ for which there exists no vertex $x \in \operatorname{Vert} P \backslash W$ with $\left\langle u_{F, v}, x\right\rangle \neq 0$ or $\left\langle u_{F, \phi(v)}, x\right\rangle \neq 0$ is at least

$$
\begin{aligned}
|\bar{A}| & -2\left((2 k+2) 2 k+(2 k+2)|C|+(k+1)|B|+\left|A^{\prime}\right|-|\bar{A}|-\eta_{-1}+d\right) \\
& =3|\bar{A}|-8(k+1) k-4(k+1)|C|-2(k+1)|B|-2\left|A^{\prime}\right|+2 \eta_{-1}-2 d .
\end{aligned}
$$

From $|\bar{A}| \geq 2\left|A^{\prime}\right|-d-2 k$ (Proposition 38) we get that this expression is at least

$$
4\left|A^{\prime}\right|-6 k-8(k+1) k-4(k+1)|C|-2(k+1)|B|+2 \eta_{-1}-5 d .
$$

Using $\left|A^{\prime}\right| \geq \eta_{-1}-|C|-|B|(k+1)$ (Proposition 32) we get that the previous expression is at least

$$
-6 k-8(k+1) k-4(k+2)|C|-3(k+1)|B|+6 \eta_{-1}-5 d .
$$

Plugging in $\eta_{-1} \geq d-2 k$ (Lemma 15 ) we deduce that the previous expression is at least

$$
-18 k-8(k+1) k-4(k+2)|C|-3(k+1)|B|+d .
$$

Finally, we use $|B|+|C| \leq 2 k$ and $|C| \leq k$ (Lemma 22) to show that the previous expression is at least

$$
d-15 k^{2}-37 k
$$

Summing up, we have proved that there are at least $d-15 k^{2}-37 k$ vertices $v \in \bar{A}$ such that all vertices $x \in \operatorname{Vert} P \backslash W$ satisfy $\left\langle u_{F, v}, x\right\rangle=0$ and $\left\langle u_{F, \phi(v)}, x\right\rangle=0$. Hence, if $d \geq 15 k^{2}-37 k+2$, then $P$ splits such that there are at least

$$
\left\lfloor\frac{d-15 k^{2}-37 k}{2}\right\rfloor \geq 1
$$

hexagons $P_{6}$ as splitting factors. This finishes the proof.

Remark 40. The reader may wonder why our proof does not achieve a linear bound. In fact, the quadratic order comes from (4), (5), and (6). These inequalities rely on several worst-case bounds such as Lemma 39. The main bottleneck at this crucial point in the proof is the separate application of these estimates when the worst cases may actually exclude each other. Hence, in order to get an overall linear bound it would be desirable to find a direct and unified approach to these estimates.

\section{Extremal smooth Fano polytopes}

In this section, we explain why Conjecture 11 and Conjecture 13 would be sharp. These conjectures were found using polymake [13]. We analyzed all smooth Fano polytopes up to dimension 9 (and checked the validity) using the database extension poly_db [15], where all the polytopes found in [17] are part of the database. 
Definition 41. Let $d=3 \cdot d^{\prime}$ for some positive integer $d^{\prime}$. Let $e_{1}, \ldots, e_{d}$ be the standard basis of $\mathbb{R}^{d}$. We define $v$ to be the following vector in $\mathbb{R}^{d}$

$$
v:=\sum_{i=1}^{d^{\prime}} e_{d^{\prime}+2 i}-\sum_{i=1}^{d^{\prime}} e_{i} .
$$

It consists of -1 in the first $d^{\prime}$ coordinates and then the coordinates alternate between 0 and 1. We define $S$ to be the simplex $S:=\operatorname{conv}\left\{v, e_{1}, e_{2}, \ldots, e_{d^{\prime}}\right\} \subseteq \mathbb{R}^{d}$. Let $P_{6} \subset \mathbb{R}^{2}$ be the hexagon from Figure 1. Finally, we define $B_{d^{\prime}}$ as the combined convex hull

$$
B_{d^{\prime}}:=\operatorname{conv}\left(S \cup\left(\{0\}^{d^{\prime}} \times P_{6}^{\oplus d^{\prime}}\right)\right) .
$$

Remark 42. Let us recall the following construction (generalizing Definition 3). Given two polytopes $Q, Q^{\prime} \subset \mathbb{R}^{d}$ whose affine hulls intersect precisely in a point (not necessarily a lattice point), then the convex hull of $Q$ and $Q^{\prime}$ is a combinatorial free sum (also called direct sum or linear join) of $Q, Q^{\prime}$. This means that its combinatorial type is dual to the product of the combinatorial dual polytope of $Q$ and the combinatorial dual polytope of $Q^{\prime}$. In particular, the combinatorial type of a combinatorial free sum of $Q$ and $Q^{\prime}$ only depends on the combinatorial types of $Q$ and $Q^{\prime}$.

Proposition 43. $B_{d^{\prime}}$ is a smooth Fano polytope of dimension $3 d^{\prime}$ with $7 d^{\prime}+1$ vertices that does not split into lower-dimensional smooth Fano polytopes.

Proof. The polytope $Q:=\{0\}^{d^{\prime}} \times P_{6}^{\oplus d^{\prime}}$ is a $2 d^{\prime}$-dimensional smooth Fano polytope (considered as $P_{6}^{\oplus d^{\prime}}$ in $\mathbb{R}^{2 d^{\prime}}$ ) with $6 d^{\prime}$ vertices. Since $S$ is a $d^{\prime}$-dimensional simplex containing $e_{1}, \ldots, e_{d^{\prime}}$, the polytope $B_{d^{\prime}}$ has clearly dimension $3 d^{\prime}$ and thus is fulldimensional. We observe that the barycenter of $S$ equals $x:=\sum_{i=1}^{d^{\prime}} \frac{1}{d^{\prime}+1} e_{d^{\prime}+2 i}$ which is a point in the interior of $Q$. This is the only intersection point of $Q$ and $S$. Hence, in the notation of Remark 42, $B_{d^{\prime}}$ is a combinatorial free sum of $Q$ and $S$. In particular, $B_{d^{\prime}}$ is simplicial and every facet $F$ of $B_{d^{\prime}}$ is the convex hull of a facet in $S$ and a facet in $Q$.

Our goal is now to show that each facet forms a lattice basis. As $P_{6}^{\oplus d^{\prime}}$ is a $2 d^{\prime}$-dimensional smooth polytope we know that $F$ contains a lattice basis for the subspace $\{0\}^{d^{\prime}} \times \mathbb{R}^{2 d^{\prime}}$. Let us distinguish whether $v \in F$ or not. Suppose $v \notin F$ then the facet contains the points $e_{1}, e_{2}, \ldots, e_{d^{\prime}}$, which is a lattice basis of the space $\mathbb{R}^{d^{\prime}} \times\{0\}^{2 d^{\prime}}$. If on the other hand $v \in F$ then one of those cartesian basis vectors is missing, let this be $e_{1}$ without loss of generality. It is sufficient to show that we can write $e_{1}$ as an integer linear combination of vertices of $F$. Consider $e_{1}=-v-\sum_{i=2}^{d^{\prime}} e_{i}+\sum_{i=1}^{d^{\prime}} e_{d^{\prime}+2 i}$. This integer linear combination consists either of vertices of $F$ or of points which can be expressed as an integer linear combination of vertices of $F$ since we already know that the vertices form a lattice basis for $\{0\}^{d^{\prime}} \times \mathbb{R}^{2 d^{\prime}}$. This shows that $B_{d^{\prime}}$ is a smooth Fano polytope.

Finally, assume that $B_{d^{\prime}}$ would decompose as a direct sum of two lower-dimensional smooth Fano polytopes $P_{1}$ and $P_{2}$. Say, $v$ is a vertex of $P_{1}$. Since $v$ is a linear combination of $e_{1}, \ldots, e_{d^{\prime}}$ and $e_{d^{\prime}+2 i}$ for $i=1, \ldots, d^{\prime}$, these vertices must also be vertices of $P_{1}$. However, no hexagon $P_{6}$ splits, so all vertices of $P$ are in $P_{1}$, a contradiction. 
Regarding the sharpness of Conjecture 11, we see that $B_{1}^{\oplus m}$ has dimension $d:=3 m$ with $8 m$ vertices. Hence, the associated toric Fano manifold has Picard number $5 m=\frac{5 d}{3}$. This example and its toric variety was already considered in [1].

Regarding the sharpness of Conjecture 13, we observe that the toric Fano manifold associated to $B_{d^{\prime}}$ has dimension $d:=3 d^{\prime}$ and Picard number $\frac{4 d+3}{3}$. We refer to the book [10] for the explanation why this defines a toric fiber bundle as stated in Conjecture 13.

Acknowledgments. The first author thanks Stockholm University for hospitality and the Berlin Mathematical School for its support. The second author is partially supported by the Vetenskapsrådet grant NT:2014-3991. We are grateful to Cinzia Casagrande for providing us with Remark 12. The authors would also like to thank the anonymous referees for several suggestions on how to improve the presentation of the paper.

\section{REFERENCES}

1. Benjamin Assarf, Michael Joswig, and Andreas Paffenholz, Smooth Fano polytopes with many vertices, Discrete \& Computational Geometry 52 (2014), no. 2, 153-194, doi: 10.1007/s00454014-9607-4.

2. Victor V. Batyrev, Toric Fano threefolds, Izv. Akad. Nauk SSSR Ser. Mat. 45 (1981), no. 4, 704-717, 927.

3. , Dual polyhedra and mirror symmetry for Calabi-Yau hypersurfaces in toric varieties, J. Algebraic Geom. 3 (1994), no. 3, 493-535, arxiv:abs/alg-geom/9310003.

4. - On the classification of toric Fano 4-folds, J. Math. Sci. (New York) 94 (1999), no. 1, 1021-1050, doi: 10.1007/BF02367245.

5. Matthias Beck, Benjamin Nill, Bruce Reznick, Carla Savage, Ivan Soprunov, and Zhiqiang Xu, Let me tell you my favorite lattice-point problem ..., Integer points in polyhedra-geometry, number theory, representation theory, algebra, optimization, statistics, Contemp. Math., vol. 452, Amer. Math. Soc., Providence, RI, 2008, doi: 10.1090/conm/452/08782, pp. 179-187.

6. Cinzia Casagrande, Toric Fano varieties and birational morphisms, International Mathematics Research Notices (2003), no. 27, 1473-1505, doi: 10.1155/S1073792803211132.

7. _ The number of vertices of a Fano polytope, Ann. Inst. Fourier (Grenoble) 56 (2006), no. 1, 121-130, doi: 10.5802/aif.2175.

8. _ On the Picard number of divisors in Fano manifolds, Ann. Sci. Éc. Norm. Supér. (4) 45 (2012), no. 3, 363-403, arxiv:abs/0905.3239.

9. __ Numerical invariants of Fano 4-folds, Math. Nachr. 286 (2013), no. 11-12, 1107-1113, 10.1002/mana.201200141.

10. David A. Cox, John B. Little, and Henry K. Schenck, Toric varieties, Graduate Studies in Mathematics, vol. 124, American Mathematical Society, Providence, RI, 2011, http: //www.cs.amherst.edu/ dac/toric.html.

11. Olivier Debarre, Fano varieties, Higher dimensional varieties and rational points (Budapest, 2001), Bolyai Soc. Math. Stud., vol. 12, Springer, Berlin, 2003, doi: 10.1007/978-3-662-051238_5, pp. 93-132.

12. Günter Ewald, Combinatorial convexity and algebraic geometry, Graduate Texts in Mathematics, vol. 168, Springer-Verlag, New York, 1996, doi: 10.1007/978-1-4612-4044-0.

13. Ewgenij Gawrilow and Michael Joswig, polymake: a framework for analyzing convex polytopes, Polytopes - combinatorics and computation (Oberwolfach, 1997), DMV Sem., vol. 29, Birkhäuser, 2000, pp. 43-73.

14. Christian Haase and Ilarion V. Melnikov, The reflexive dimension of a lattice polytope, Ann. Comb. 10 (2006), no. 2, 211-217, doi: 10.1007/s00026-006-0283-9.

15. Silke Horn, poly_db database extension, http://solros.de/polymake/poly_db/.

16. Maximilian Kreuzer and Benjamin Nill, Classification of toric Fano 5-folds, Adv. Geom. 9 (2009), no. 1, 85-97, doi: 10.1515/ADVGEOM.2009.005.

17. Benjamin Lorenz and Andreas Paffenholz, Smooth reflexive polytopes up to dimension 9, 2008, http://polymake.org/polytopes/paffenholz/www/fano.html. 
18. Benjamin Nill, Gorenstein toric Fano varieties, Manuscr. Math. 116 (2005), no. 2, 183-210, doi: 10.1007/s00229-004-0532-3.

19. Benjamin Nill and Alexander Kasprzyk, Fano polytopes, in: Strings, Gauge Fields, and the Geometry Behind - The Legacy of Maximilian Kreuzer, World Scientific, 2012, available at http://magma.maths.usyd.edu.au/users/kasprzyk/research/pdf/fano_polytopes .pdf.

20. Benjamin Nill and Mikkel Øbro, $\mathbb{Q}$-factorial Gorenstein toric Fano varieties with large Picard number, Tohoku Math. J. (2) 62 (2010), no. 1, 1-15, doi: 10.2748/tmj/1270041023.

21. Mikkel Øbro, An algorithm for the classification of smooth Fano polytopes, 2007, preprint, arxiv:0704.0049.

22. _ Classification of smooth Fano polytopes, Ph.D. thesis, University of Aarhus, 2007, available at https://pure.au.dk/portal/files/41742384/imf_phd_2008_moe.pdf.

23. _ Classification of terminal simplicial reflexive d-polytopes with $3 d-1$ vertices, Manuscripta Math. 125 (2008), no. 1, 69-79, doi: 10.1007/s00229-007-0133-z.

24. Hiroshi Sato, Toward the classification of higher-dimensional toric Fano varieties, Tohoku Math. J. (2) 52 (2000), no. 3, 383-413, doi: 10.2748/tmj/1178207820.

25. Keiichi Watanabe and Masayuki Watanabe, The classification of Fano 3-folds with torus embeddings, Tokyo J. Math. 5 (1982), no. 1, 37-48, doi: 10.3836/tjm/1270215033.

BENJAMIN ASSARF

TU-Berlin, Str. Des 17. Juni 136, D-10623 Berlin, Germany

E-mail address: assarf@math.tu-berlin.de

BENJAMIN NILL

Stockholm University, Kräftriket, Se-10691 Stockholm, Sweden

E-mail address: nill@math.su.se 